\title{
STRONG HOMOLOGY AND THE PROPER FORCING AXIOM
}

\author{
ALAN DOW, PETR SIMON, AND JERRY E. VAUGHAN
}

(Communicated by Dennis Burke)

\begin{abstract}
This paper concerns applications of set theory to the problem of calculating the strong homology of certain subsets of Euclidean spaces. We prove the set theoretic result that it is consistent that every almost coinciding family indexed by ${ }^{\omega} \omega$ is trivial (e.g., the proper forcing axiom implies this). This result, combined with results of S. Mardešić and A. Prasalov, show that the statement "the $k$-dimensional strong homology of $Y^{(k+1)}$ (the discrete sum of countably many copies of the $(k+1)$-dimensional Hawaiian earring) is trivial" is consistent with and independent of the usual axioms of set theory.
\end{abstract}

\section{INTRODUCTION}

In Euclidean space $\mathbf{R}^{k+1}$, let $C_{n}$ be the $k$-sphere with center $(1 / n, 0, \ldots, 0)$ and radius $1 / n$ for $n \geq 1$, and let $X^{(k)}=\bigcup\left\{C_{n}: n \geq 1\right\} ; X^{(k)}$ is called the $k$-dimensional Hawaiian earring. Let $\mathscr{F}(k)$ be a family consisting of countably many copies of $X^{(k)}$, and let $Y^{(k)}$ denote the topological (discrete) sum of $\mathscr{F}(k)$. S. Mardešić and A. Prasolov [MP] translated the calculation of the $k$-dimensional strong homology of $Y^{(k+1)}$ into a condition in set theory. (Theorem 1.1), and it is this condition which we consider in the remainder of this paper. The calculation of the strong homology of these spaces is of interest in the question of whether strong homology satisfies the additivity axiom (of J. Milnor [M]). Let ${ }^{\omega} \omega=\{f: f: \omega \rightarrow \omega\}$ denote the set of all functions from the set $\omega$ of natural numbers into $\omega$.

1.1. Theorem [MP]. The k-dimensional strong homology of $Y^{(k+1)}$ is nontrivial if and only if there exists a collection of functions $\left\{g_{f}: f \in{ }^{\omega} \omega\right\}$ such that

(i) for all $f \in{ }^{\omega} \omega, \operatorname{dom}\left(g_{f}\right)=\{(n, m): m \leq f(n)\}$, and $\operatorname{range}\left(g_{f}\right) \subset \omega$,

(ii) for any pair $f, f^{\prime},\left\{x \in \operatorname{dom}\left(g_{f}\right) \cap \operatorname{dom}\left(g_{f^{\prime}}\right): g_{f}(x) \neq g_{f^{\prime}}(x)\right\}$ is finite, and

(iii) there does not exist a function $g: \omega \times \omega \rightarrow \omega$ such that for every $f$, $\left\{x \in \operatorname{dom}\left(g_{f}\right): g(x) \neq g_{f}(x)\right\}$ is finite.

Received by the editors May 17, 1988 and, in revised form September 16, 1988.

1980 Mathematics Subject Classification (1985 Revision). Primary 55N07, 03E35.

Key words and phrases. Strong homology, Hawaiian earring, almost coinciding families, continuum hypothesis, bounding number, dominating number, $(\kappa, \kappa)$-gaps in the sense of Hausdorff, $\aleph_{1}$-scale. 
We consider [MP, Question 5]: Does there exist a family of functions satisfying the three conditions in Theorem 1.1?

The authors of the present paper worked at first independently on different aspects of this question. This joint paper is the result of the author's discussions at STACY (Set Theory and its Applications Conference at York University, August, 1987). Other people at the conference also took part in these discussions, especially Stevo Todorc̀ević, who conceived the proof given in $\S 4$. We also thank the referee for several helpful suggestions.

In [MP, Theorem 2] there is a proof showing that under the assumption of the continuum hypothesis $(\mathrm{CH})$, the answer to their Question 5 is "yes". Consequently, $\mathrm{CH}$ implies that the $k$-dimensional strong homology of $Y^{(k+1)}$ is nontrivial. We improve this slightly by showing that the continuum hypothesis $\left(c=\aleph_{1}\right)$ in the statement of that result can be replaced by the weaker hypothesis "d $=\aleph_{1} "$ (see Theorem 2.4).

The main purpose of this paper is to prove

1.2. Theorem. It is consistent with the usual axiom of set theory that there does not exist a family of functions satisfying the three conditions in 1.1 , and therefore the $k$-dimensional strong homology of $Y^{(k+1)}$ is trivial. Thus, whether or not the $k$-dimensional strong homology of $Y^{(k+1)}$ is trivial, depends on set theory.

We give two different proofs of Theorem 1.2 (see $\S 3$ and $\S 4$ ). In $\S 4$ we prove Theorem 1.2 by showing that it reduces to a problem about gaps which was solved by K. Kunen about ten years ago, but never published (see [B, p. 957] for a brief outline of Kunen's construction). This reduction of Theorem 1.2 was pointed out to us by Stevo Todorčević, and is included here with his permission. The second proof of Theorem 1.2 (given in $\S 3$ ) is a standard application of the proper forcing axiom. We include this proof as another example of how the axiom can be applied. The consistency of the proper forcing axiom requires a large cardinal assumption, and often that assumption can be removed from specific applications by standard techniques (cf. [B, p. 957]). This is true in this case, but Kunen's proof already demonstrates that no large cardinals are needed for the proof of Theorem 1.2.

\section{Definitions AND “ $\mathfrak{d}=\aleph_{1} "$}

Let $\omega$ denote the set of natural numbers, and ${ }^{\omega} \omega$ denote the set of all functions $f$ with domain $\omega$, and range (a subset of) $\omega$. Let $f, g \in{ }^{\omega} \omega$. The mod finite order $\leq^{*}$ on ${ }^{\omega} \omega$ is defined by $f \leq^{*} g$ provided there exists $N \in \omega$ such that $f(n) \leq g(n)$ for all $n \geq N$. We say $f<^{*} g$ if there exists $N \in \omega$ such that $f(n)<g(n)$ for all $n \geq N$. In the mod finite order, the constant function with value zero is the smallest element. Thus, we say that a set is bounded in the mod finite order provided it has an upper bound. We recall the 
well-known fact that every countable set $A \subset{ }^{\omega} \omega$ is bounded in the mod finite order.

The cardinal number $\mathfrak{b}$, the bounding number, is defined as follows:

$$
\mathfrak{b}=\min \left\{|U|: U \subset{ }^{\omega} \omega \text { is not bounded in the } \bmod \text { finite order }\right\} .
$$

A subset $D$ of $I \subset{ }^{\omega} \omega$ is said to be dominating in $I$ (or cofinal in $I$ ) with respect to the mod finite order if for every $g \in I$ there exists $f \in D$ such that $g \leq^{*} f$. Clearly, if $D$ is dominating in ${ }^{\omega} \omega$ and $g \in{ }^{\omega} \omega$ then there exists $h$ in $D$ such that $g<^{*} h$.

The cardinal $\mathfrak{d}$, the dominating number, is defined as follows:

$$
\mathfrak{d}=\min \left\{|D|: D \subset{ }^{\omega} \omega \text { is dominating in }{ }^{\omega} \omega\right\} .
$$

We denote the cardinality of the continuum by $\mathfrak{c}$. Obviously, $\aleph_{1} \leq \mathfrak{b} \leq \mathfrak{d} \leq \mathfrak{c}$. An ordered family $S=\left\{f_{\alpha}: \alpha<\kappa\right\}$, where $\kappa$ is a cardinal number, is called a $\kappa$-scale provided for every $\alpha<\beta<\kappa, f_{\alpha}<^{*} f_{\beta}$, and $S$ is dominating in ${ }^{\omega} \omega$. It is easy to see that there exists a $\kappa$-scale if and only if $\mathfrak{b}=\mathfrak{d}=\kappa$. In particular, " $d=\aleph_{1}$ " is equivalent to the existence of an $\aleph_{1}$-scale. For a discussion of these cardinals, see [vD].

For $f \in{ }^{\omega} \omega$, let $L_{f}$ denote the lower set of points with respect to the graph of $f$, i.e., $L_{f}=\{(i, j) \in \omega \times \omega: j \leq f(i)\}$. For simplicity, if we have an indexed function, say $f_{\alpha}$, we write $L_{\alpha}$ instead of $L_{f_{n}}$.

Let $I \subset{ }^{\omega} \omega$. An almost coinciding family indexed by $I$ is a family of functions $\left\{g_{f}: f \in I\right\}$ such that (1) $g_{f}: L_{f}: \rightarrow \omega$ for all $f \in I$, and (2) for all $f, h \in I, \quad\left\{x \in L_{f} \cap L_{h}: g_{f}(x) \neq g_{h}(x)\right\}$ is finite (for short, we write $g_{f}={ }^{*} g_{h}$ ). An almost coinciding family indexed by $I$ is called trivial if there exists $g: \omega \times \omega \rightarrow \omega$ such that $g={ }^{*} g_{f}$ on $L_{f}$ for all $f \in{ }^{\omega} \omega$. For simplicity, if we have an indexed function, say $f_{\alpha} \in{ }^{\omega} \omega$, we will write $g_{\alpha}$ instead of $g_{f_{n}}$.

The question of Mardešic and Prasolov can now be stated as follows: Does there exist a nontrivial almost coinciding family indexed by ${ }^{\omega} \omega$ ?

The following lemmas are implicit in the proof of Theorem 2 in [MP], and the proofs are left to the reader.

2.2. Lemma. Every almost coinciding family with a countable index set is trivial.

2.2. Lemma. Every almost coinciding family indexed by $I$ can be extended to an almost coinciding family indexed by the set of all functions which are dominated (in the mod finite order) by $I$, i.e. to the index set $J=\{g \in$ $\left.{ }^{\omega} \omega:(\exists f \in I)\left(g \leq^{*} f\right)\right\}$.

2.3. Corollary. Let $I \subset{ }^{\omega} \omega$. There exists a nontrivial, almost coinciding family indexed by $I$ if and only if there exists a nontrivial, almost coinciding family indexed by a dominating (mod finite) subset of $I$.

2.4. Theorem. If $\mathfrak{d}=\aleph_{1}$, (i.e., there exists an $\aleph_{1}$-scale) then there exists a nontrivial, almost coinciding family indexed by ${ }^{\omega} \omega$. 
Proof. Let $\left\{f_{\alpha}: \alpha<\omega_{1}\right\} \subset{ }^{\omega} \omega$ be an $\aleph_{1}$-scale of strictly increasing functions (i.e., each $f_{\alpha}$ is strictly increasing on $\omega$ ). Let $\varphi: \omega \times \omega \rightarrow \omega$ be a bijection. Assume we have constructed $g_{\alpha}$ for $\alpha<\gamma$ (where $\gamma<\omega_{1}$ ) such that

(1) $g_{\alpha}: L_{\alpha} \rightarrow \omega$

(2) if $\beta<\alpha$ then $g_{\alpha}={ }^{*} g_{\beta}$ on $L_{\alpha} \cap L_{\beta}$,

(3) for all $x \in \operatorname{graph}\left(f_{\alpha}\right), g_{\alpha}(x)=f_{\alpha} \circ \varphi(x)$.

Construct $g_{\gamma}$ as follows. By Lemma 1.1, the countable almost coinciding family $\left\{g_{\alpha}: \alpha<\gamma\right\}$ is trivial; so there exists $g: \omega \times \omega \rightarrow \omega$ such that $g={ }^{*} g_{\alpha}$ on $L_{\alpha}$ for all $\alpha<\gamma$. For every $x \in L_{\gamma}$ define

$$
g_{\gamma}(x)= \begin{cases}g(x) & \text { if } x \in L_{\gamma}-\operatorname{graph}\left(f_{\gamma}\right) \\ f_{\gamma} \circ \varphi(x) & \text { if } x \in \operatorname{graph}\left(f_{\gamma}\right) .\end{cases}
$$

Since for each $\alpha<\gamma, f_{\alpha}<^{*} f_{\gamma}$, it follows that $\left\{g_{\alpha}: \alpha \leq \gamma\right\}$ is an almost coinciding family satisfying (3) above. This completes the induction, and we have $\left\{g_{\alpha}: \alpha<\omega_{1}\right\}$, which is obviously an almost coinciding family indexed by $\left\{f_{\alpha}: \alpha<\omega_{1}\right\}$. We need to show that it is nontrivial. If it is trivial, then there exists $g: \omega \times \omega \rightarrow \omega$ such that $g={ }^{*} g_{\alpha}$ on $L_{\alpha}$ for all $\alpha<\omega_{1}$. Since $\left\{f_{\alpha}: \alpha<\right.$ $\left.\omega_{1}\right\}$ is dominating, there exists $f_{\alpha}$ such that $g \circ \varphi^{-1}<^{*} f_{\alpha}$. Thus there exists $N \in \omega$ such that for all $x \in \varphi^{-1}(\omega-N) \cap \operatorname{graph}\left(f_{\alpha}\right)$ we have $g \circ \varphi^{-1}(\varphi(x))<$ $f_{\alpha}(\varphi(x))$ and $g(x)=g_{\alpha}(x)$; so by definition of $g_{\alpha}$, we have $f_{\alpha}(\varphi(x))<$ $f_{\alpha}(\varphi(x))$ which is a contradiction. Thus, $\left\{g_{\alpha}: \alpha<\omega_{1}\right\}$ is a nontrivial, almost coinciding family; so by Corollary 2.3 this completes the proof.

\section{THE PROPER FORCING AXIOM (PFA) AND ALMOST COINCIDING FAMILIES}

In this section we prove Theorem 1.2, which we restate as follows.

3.1. Theorem. The proper forcing axiom implies that every almost coinciding family indexed by ${ }^{\omega} \omega$ is trivial.

Proof. (By contradiction). We will need the following three lemmas, and we assume for them that $\left\{g_{f}: f \in{ }^{\omega} \omega\right\}$ is a nontrivial, almost coinciding family.

3.2 Lemma. If $A \subset{ }^{\omega} \omega$ is uncountable, and for each distinct pair $f, f^{\prime} \in A$ there exists $x \in L_{f} \cap L_{f^{\prime}}$ such that $g_{f}(x) \neq g_{f^{\prime}}(x)$, then $A$ is unbounded in the mod finite order on ${ }^{\omega} \omega$.

Proof. Suppose that $A$ is bounded by a function $f_{0} \in{ }^{\omega} \omega$. For each $f$ in $A$, there exists $N_{f} \in \omega$ such that $f(n) \leq f_{0}(n)$ for all $n \geq N_{f}$, and $g_{f}(x)=g_{0}(x)$ for all $x \in L_{f}-\left(N_{f} \times \omega\right)$. Since $A$ is uncountable, there exists $N \in \omega$ and an uncountable $B \subset A$ such that $N_{f}=N$ for all $f \in B$. Since $N \times \omega$ is countable, there exists a finite $F \subset N \times \omega$ and an uncountable $C \subset B$ such that $L_{f} \cap N \times \omega=F$ for all $f \in C$. Since there are only countably many functions from $F$ into $\omega$ there exists $\sigma: F \rightarrow \omega$ and an uncountable $D \subset C$ such that $g_{f} \mid F=\sigma$ for all $f \in D$. Now it follows that if $f, f^{\prime}$ are 
distinct elements of $D$, then $g_{f}$ and $g_{f^{\prime}}$ agree on $L_{f} \cap L_{f^{\prime}}$, but this contradicts the hypothesis.

3.3. Lemma. If $D \subset{ }^{\omega} \omega$ is dominating in the mod finite order, and there exists $N \in \omega$ such that for all $f, f^{\prime} \in D$, and for all $x \in L_{f} \cap L_{f^{\prime}}-(N \times \omega)$ we have $g_{f}(x)=g_{f^{\prime}}(x)$, then the almost coinciding family $\left\{g_{f}: f \in D\right\}$ is trivial.

Proof. We are given that $g=\cup\left\{g_{f} \mid\left(L_{f}-(N \times \omega)\right): f \in D\right\}$ is a function. We must show that $\operatorname{dom}(g) \supseteq(\omega-M) \times \omega$ for some $M \geq N$. If no such $M$ exists then we can construct a strictly increasing sequence $\left(n_{i}\right)$ in $\omega$ and integers $m_{i}$ such that for all $i \in \omega,\left(n_{i}, m_{i}\right) \notin \cup\left\{L_{f}: f \in D\right\}$. But $\left\{\left(n_{i}, m_{i}\right): i \in \omega\right\}$ can be extended to a function $h$ with domain $\omega$, and since $D$ is dominating, there exists $f \in D$ such that $h \leq^{*} f$. This implies that there are (infinitely many) $i$ such that $m_{i} \leq f\left(n_{i}\right)$ which contradicts the definition of $m_{i}$.

For a set $X$, let $[X]^{<\omega}$ denote the set of all finite subsets of $X$.

Lemma 3.4. If $S$ is an $\aleph_{1}$-scale in ${ }^{\omega} \omega$ and $\left\{g_{f}: f \in S\right\}$ is a nontrivial almost coinciding family, then the poset

$$
\begin{aligned}
& Q(S)=\left\{s \in[S]^{<\omega}: \text { if } f \neq f^{\prime}\right. \text { in s then } \\
& \left.\qquad \exists x \in(\omega \times \omega)\left(x \in L_{f} \cap L_{f^{\prime}} \text { and } g_{f}(x) \neq g_{f^{\prime}}(x)\right)\right\}
\end{aligned}
$$

ordered by reverse inclusion (i.e., $s$ extends $s^{\prime}\left(s \leq s^{\prime}\right)$ iff $\left.s \supset s^{\prime}\right)$ satisfies the CCC.

Proof. Suppose that $\left\{s_{\alpha}: \alpha<\omega_{1}\right\}$ is an antichain in $Q(S)$. By the $\Delta$-system lemma, we may assume that the $s_{\alpha}$ are pairwise disjoint. Since $s_{\alpha}$ is finite and linearly ordered by the mod finite order on ${ }^{\omega} \omega$, we may let $f_{\alpha}=\min \left(s_{\alpha}\right)$. For each $\alpha<\omega_{1}$ there exists $N_{\alpha} \in \omega$ such that for all $n \geq N_{\alpha}$

(1) for all $f \in s_{\alpha}, f_{\alpha}(n) \leq f(n)$, and

(2) for all $x \in L_{f} \cap L_{\alpha}-\left(N_{\alpha} \times \omega\right), g_{f}(x)=g_{\alpha}(x)$.

There exists an uncountable $A \subset \omega_{1}$ and $N \in \omega$ such that $N_{\alpha}=N$ for all $\alpha \in A$. We now claim that $\left\{f_{\alpha}: \alpha \in A\right\}$ and $\left\{g_{\alpha}: \alpha \in A\right\}$ satisfy the hypothesis of Lemma 3.3. To see this, let $\alpha<\beta$ in $A$. Since $s_{\alpha}$ and $s_{\beta}$ are incompatible in $Q(S)$, there exist $f \in s_{\alpha}$ and $f^{\prime} \in s_{\beta}$ such that for all $x \in L_{f} \cap L_{f^{\prime}}$, $g_{f}(x)=g_{f^{\prime}}(x)$. Thus for all $x \in L_{\alpha} \cap L_{\beta}-(N \times \omega)$ we have $g_{f}(x)=g_{\alpha}(x)$ and $g_{f^{\prime}}(x)=g_{\beta}(x)$; so $g_{\alpha}(x)=g_{\beta}(x)$. Thus by Lemma 3.3, $\left\{g_{\alpha}: \alpha \in A\right\}$ is trivial, but by Corollary 2.3, this implies $\left\{g_{f}: f \in S\right\}$ is trivial. This contradicts our hypothesis.

Proof of 3.1. Recall that we have assumed there is a fixed nontrivial, almost coinciding family $\left\{g_{f}: f \in{ }^{\omega} \omega\right\}$. We assume PFA; hence $\operatorname{MA}\left(\aleph_{1}\right)$ holds; so $\aleph_{2} \leq \mathfrak{b} \leq \mathfrak{c}$. We will use PFA in the form of the cardinal-collapsing trick (see [B, p. 924]), and thus we start by letting $P$ be a countably closed poset which collapses $\mathfrak{c}$ to $\omega_{1}$. In the generic extension $V^{P}, \omega_{1}$ is preserved, no new functions from $\omega$ to $\omega$ are added (i.e., $\left.\left({ }^{\omega} \omega\right)^{V^{P}}=\left({ }^{\omega} \omega\right)^{V}\right)$, and the continuum 
hypothesis holds; so there exists an $\aleph_{1}$-scale in ${ }^{\omega} \omega$. Let $S$ be an $\aleph_{1}$-scale $\left(S \in V^{P}\right)$. Since countably closed forcing adds no new functions from $(\omega \times \omega)$ into $\omega$, the corresponding almost coinciding family $\left\{g_{f}: f \in S\right\}$ is nontrivial. Working in $V^{P}$, we denote $S$ by $\left\{f_{\alpha}: \alpha<\omega_{1}\right\}$ so that for $\alpha<\beta<\omega_{1}$ we have $f_{\alpha}<^{*} f_{\beta}$. For each $\alpha<\omega_{1}$ let $Q_{\alpha}$ be the poset associated with the $\aleph_{1}$-scale $\left\{f_{\beta}: \alpha<\beta<\omega_{1}\right\}$, where we consider elements of $Q_{\alpha}$ to be finite subsets of $\omega_{1}$. By Lemma 3.4, each $Q_{\alpha}$ satisfies the CCC. We need some $Q_{\alpha}$ to force an uncountable subset of $\omega_{1}$; so by the standard density argument, we need to show: there exists $\alpha<\omega_{1}$ such that for every $\beta>\alpha$

$$
D(\beta)=\left\{s \in Q_{\alpha}:(\exists \gamma>\beta) \gamma \in s\right\}
$$

is dense in $Q_{\alpha}$. Suppose to the contrary that for all $\alpha<\omega_{1}$ there exists $\beta>\alpha$ such that $D(\beta)$ is not dense in $Q_{\alpha}$. Then for all $\alpha<\omega_{1}$ there exist $s_{\alpha} \in Q_{\alpha}$ and $\beta_{\alpha}>\alpha$ such that for all $\gamma>\beta_{\alpha}, s_{\alpha} \cup\{\gamma\} \notin Q_{\alpha}$. Let $\mu(\alpha)=\min \left(s_{\alpha}\right)$, and choose $N_{\alpha}$ so that

(1) for all $\tau \in s_{\alpha}$ and $n \geq N_{\alpha}, f_{\mu(\alpha)}(n) \leq f_{\tau}(n)$, and

(2) for all $x \in L_{\tau} \cap L_{\mu(\alpha)}-\left(N_{\alpha} \times \omega\right), g_{\tau}(x)=g_{\mu(\alpha)}(x)$.

Since $s_{\alpha} \cup\{\gamma\} \notin Q_{\alpha}$, there exists $\tau=\tau(\gamma) \in s_{\alpha}$ such that $g_{\gamma}(x)=g_{\tau}(x)$ for all $x \in L_{\gamma} \cap L_{\tau}$, and this implies that $g_{\gamma}(x)=g_{\mu(\alpha)}(x)$ for all $x \in L_{\gamma} \cap L_{\mu(\alpha)}$ $\left(N_{\alpha} \times \omega\right)$. Let $B$ be an uncountable subset of $\omega_{1}$ such that if $\alpha<\beta$ in $B$, then $\mu(\alpha)<\mu(\beta)$, and $\beta_{\alpha}<\mu(\beta)$. There exists $N \in \omega$ and an uncountable $A \subset B$ such that $N_{\alpha}=N$ for all $\alpha$ in $A$. Hence, for all $\alpha<\beta$ in $A$, we have $g_{\mu(\alpha)}(x)=g_{\mu(\beta)}(x)$ for all $x \in L_{\mu(\alpha)} \cap L_{\mu(\beta)}-(N \times \omega)$. It follows from Lemma 3.3 that the almost coinciding family $\left\{g_{\mu(\alpha)}: \alpha \in A\right\}$ is trivial. But by Corollary 2.3, this implies that $\left\{g_{\alpha}: \alpha<\omega_{1}\right\}$ is trivial, which is a contradiction. Thus for some $\alpha$, every $D(\beta)$ is dense in $Q_{\alpha}$. Let $Q$ denote such a $Q_{\alpha}$. Thus forcing with $Q$ adds an uncountable subset of $\omega_{1}$, hence an uncountable set $T \subset S$ such that for distinct $f, f^{\prime}$ in $T$, there exists $x \in L_{f} \cap L_{f^{\prime}}$ such that $g_{f}(x) \neq g_{f^{\prime}}(x)$. In $V^{P * Q}$, there is a bijection $F: \omega_{1} \rightarrow T \subset S \subset\left({ }^{\omega} \omega\right)^{V}$. We will apply PFA to $P * Q$; so with that in mind we note that there are $P * Q$-names $\Phi$ and $t$ such that

(1) $1 \Vdash \Phi:\left(\omega_{1}\right)^{\vee} \rightarrow t$ is a bijection and $t \subset\left({ }^{\omega} \omega\right)^{\vee}$,

(2) $1 \Vdash$ (for all $\left.f, f^{\prime} \in\left({ }^{\omega} \omega\right)^{\vee}\right)$ (if $f, f^{\prime} \in t$ and $f \neq f^{\prime}$, then $\exists x \in\left({ }^{\omega} \omega\right)^{\vee}$ ) $\left(x \in \stackrel{v}{L}_{f} \cap \stackrel{\vee}{L}_{f^{\prime}}\right.$ and $\left.\stackrel{\vee}{g}_{f}(x) \neq \stackrel{\vee}{g}_{f^{\prime}}(x)\right)$. (We now use standard abuse of notation for the forcing language.) For every $\alpha<\omega_{1}$ let

$$
D(\alpha)=\left\{r \in P * Q:\left(\exists f \in{ }^{\omega} \omega\right) r \Vdash \Phi(\alpha)=f\right\} .
$$

By (1), each $D(\alpha)$ is dense in $P * Q$, and since there are only $\aleph_{1}$ of them, by PFA there exists (in $V$ ) a filter $G \subset P * Q$ such that $G \cap D(\alpha) \neq \varnothing$ for all $\alpha<\omega_{1}$. Now we work in $V$. For each $\alpha<\omega_{1}$, pick $r_{\alpha} \in G$ and $f_{\alpha} \in{ }^{\omega} \omega$ such that $r_{\alpha} \Vdash \Phi(\alpha)=f_{\alpha}$. Put

$$
Z=\left\{f_{\alpha}: \alpha<\omega_{1}\right\} .
$$


We claim that if $\alpha<\beta<\omega_{1}$, then $f_{\alpha} \neq f_{\beta}$. To see this, note that there exists $r \in G$, such that $r$ extends both $r_{\alpha}$ and $r_{\beta}$, and thus $r \Vdash " \Phi(\alpha)=f_{\alpha}$ and $\Phi(\beta)=f_{\beta}$." Since $\alpha<\beta$ and by (1), $1 \Vdash$ " $\Phi$ is one-one", we have $r \Vdash$ " $f_{\alpha} \neq f_{\beta}$ ". Thus by absoluteness, $f_{\alpha} \neq f_{\beta}$; so in particular $|Z|=\aleph_{1}$. Now we claim that if $f$ and $f^{\prime}$ are distinct elements of $Z$, then there exists $x \in(\omega \times \omega)$ such that $g_{f}(x) \neq g_{f^{\prime}}(x)$. To see this, let $\alpha<\beta<\omega_{1}$, and as before choose $r \in G$ so that $r \Vdash " \Phi(\alpha)=f_{\alpha}$ and $\Phi(\beta)=f_{\beta}$ ". By (1) we know that $1 \Vdash$ "range $(\Phi) \subset t$ "; so we have $r \Vdash$ " $f_{\alpha}, f_{\beta} \in t$ and $f_{\alpha} \neq f_{\beta}$ ". Hence by (2) we get

$$
r \Vdash(\exists x \in \omega \times \omega)\left(g_{\alpha}(x) \neq g_{\beta}(x)\right) .
$$

Thus there exist $s \in P * Q$, which extends $r$, and $x \in \omega \times \omega$ such that $s \Vdash$ “ $g_{\alpha}(x) \neq g_{\beta}(x)$ "; so by absoluteness, $g_{\alpha}(x) \neq g_{\beta}(x)$.

Now we have proved that $Z$ is uncountable and satisfies the hypothesis of Lemma 3.2; so $Z$ is unbounded in the mod finite order on ${ }^{\omega} \omega$. On the other hand, PFA implies $\mathfrak{b} \geq \aleph_{2}$; so we have $|Z| \geq \aleph_{2}$, and that contradicts the fact that $|Z|=\aleph_{1}$. This completes the proof.

\section{Almost COINCIDING FAMILIES AND $(\kappa, \kappa)$-GAPS}

In this section we give the proof of Theorem 1.2 due to Stevo Todorčević.

We recall some definitions concerning infinite subsets of the natural numbers. Let $[\omega]^{\omega}=\{X \subset \omega: X$ is infinite $\}$. For $X, Y \in[\omega]^{\omega}$ we say $X \subset^{*} Y$ provided $X-Y$ is finite. A family $\left\{\left(A_{\alpha}, B_{\alpha}\right): \alpha<\kappa\right\}$ is called a $(\kappa, \kappa)$-gap provided (i) for all $\alpha<\beta<\kappa, A_{\alpha} \subset^{*} A_{\beta}$ and $B_{\alpha} \subset^{*} B_{\beta}$, (ii) $A_{\alpha} \cap B_{\beta}$ is finite for all $\alpha, \beta<\kappa$, and (iii) there does not exist a set $E \in[\omega]^{\omega}$ such that $B_{\alpha} \cap E$ is finite and $A_{\alpha} \subset^{*} E$ for all $\alpha<\kappa$.

4.1. Theorem. If there exists an unbounded chain $S=\left\{f_{\alpha}: \alpha<\kappa\right\} \subset{ }^{\omega} \omega$ consisting of nondecreasing functions, and also a nontrivial, almost coinciding family $\left\{g_{\alpha}: \alpha<\kappa\right\}$ indexed by $S$, then there exists $a(\kappa, \kappa)$-gap.

Proof. Let $S=\left\{f_{\alpha}: \alpha<\kappa\right\}$ be as stated and $\left\{g_{\alpha}: \alpha<\kappa\right\}$ an associated nontrivial almost coinciding family. It suffices to construct the gap on the countable set $\omega \times \omega \times \omega$. Let

$$
A_{\alpha}=\left\{(n, m, i):(n, m) \in L_{\alpha} \text { and } i=g_{\alpha}(n, m)\right\},
$$

and

$$
B_{\alpha}=\left\{(n, m, i):(n, m) \in L_{\alpha}, \text { and } i<g_{\alpha}(n, m)\right\} \text {. }
$$

First we check that if $\alpha<\beta<\kappa$ then $A_{\alpha} \subset^{*} A_{\beta}$. Choose $N$ so large that for all $(n, m) \in \omega \times \omega-N \times N$, if $(n, m) \in L_{\alpha}$, then $(n, m) \in L_{\beta}$ and $g_{\alpha}(n, m)=$ $g_{\beta}(n, m)$. Let $M=\max \left\{g_{\alpha}(s, t): s, t \leq N\right\}+1$. Then $A_{\alpha}-N \times N \times M \subset A_{\beta}$. The proofs that $B_{\alpha} \subset^{*} B_{\beta}$ and that $A_{\alpha} \cap B_{\beta}$ is finite are similar. It only remains 
to check that there is no set that "fills the gap." Suppose that $E$ fills the gap, i.e., $A_{\alpha} \subset^{*} E$ and $B_{\alpha} \cap E$ is finite for all $\alpha<\kappa$. Define $g: \omega \times \omega \rightarrow \omega$ by

$$
g(n, m)= \begin{cases}\min \{i:(n, m, i) \in E\} & \text { if possible } \\ 0 & \text { otherwise }\end{cases}
$$

If $N$ is such that $A_{\alpha}-E, B_{\alpha}-E \subset N \times \omega^{2}$, then $g$ and $g_{\alpha}$ agree beyond $N$. This, however, contradicts the assumption that the almost coinciding family was nontrivial. Thus $\left\{\left(A_{\alpha}, B_{\alpha}\right): \alpha<\kappa\right\}$ is a $(\kappa, \kappa)$-gap.

4.2. Lemma (K. Kunen (unpublished; $\mathrm{cf}$. [B, 4.3, p. 957])). It is consistent to assume that $\mathfrak{c}=\mathfrak{b}=\aleph_{2}$, and there does not exist a (c, $\mathfrak{c}$ )-gap.

We also need the following easy lemma.

4.3. Lemma. If $\left\{g_{f}: f \in{ }^{\omega} \omega\right\}$ is a nontrivial almost coinciding family indexed by ${ }^{\omega} \omega$, and $S \subset{ }^{\omega} \omega$ is unbounded in the mod finite order, then $\left\{g_{f}: f \in S\right\}$ is nontrivial.

Proof that every almost coinciding family indexed by ${ }^{\omega} \omega$ is trivial in Kunen's model 4.2: By definition of $\mathfrak{b}$, it is straightforward to construct an unbounded chain $S \subset{ }^{\omega} \omega$ of cardinality $\mathfrak{b}$ consisting of nondecreasing functions. If there exists a nontrivial almost coinciding family indexed by ${ }^{\omega} \omega$, then by Lemmas 4.3 , and 4.1 , there exists a $(\mathfrak{b}, \mathfrak{b})$-gap. By Theorem 4.2 , this is impossible in Kunen's model.

The following problem is motivated by 4.1 and 2.4 .

Problem. Is it consistent that if there exist an unbounded chain $S \subset{ }^{\omega} \omega$ consisting of nondecreasing functions (or a $\kappa$-scale) and a $(\kappa, \kappa)$-gap. Then there exists a nontrivial, almost coinciding family indexed by ${ }^{\omega} \omega$ ?

\section{REFERENCES}

[B] James E. Baumgartner, Applications of proper forcing, Handbook of Set-theoretic Topology, (K. Kunen and J. Vaughan, eds.), North-Holland, Amsterdam, 1984, pp. 913-959.

[vD] Eric K. van Douwen, The integers and topology, Handbook of Set-theoretic Topology, (K. Kunen and J. Vaughan, eds.) North-Holland, Amsterdam, 1984, pp. 111-167.

[LM] Ju. T. Lisica and S. Mardešić, Strong homology of inverse systems. III, Topology and its Appl. 20 (1985), 29-37.

[M] J. Milnor, On axiomatic homology theory, Pacific J. Math. 12 (1962), 337-341.

[MP] S. Mardešić and A. Prasolov, Strong homology is not additive, Trans. Amer. Math. Soc. 307 (1988), 725-744.

Department of Mathematics, York University, North York, Ontario, Canada M3J 1 P3

Matematický Ústav University Karlov, Sokolovská 83,18600 Praha 8, Czechoslovakia

Department of Mathematics, University of North Carolina at Greensboro, Greensboro, North Carolina 27412 